\title{
Euler Formula Derivation
}

\author{
Wikaria Gazali \\ Mathematics \& Statistics Department, School of Computer Science, \\ Bina Nusantara University, \\ Jakarta, Indonesia 11480 \\ wikaria@binus.ac.id
}

*Correspondence: wikaria@binus.ac.id

\begin{abstract}
This paper discusses the derivation of Euler's formula. To obtain this model, the writer derives Euler's formula from $\mathrm{e}^{\mathrm{x}+\mathrm{i} y}$ by first finding the norm and argument of $\mathrm{e}^{\mathrm{x}+\mathrm{i}}$. In this derivation we substitute the norm and argument of $\mathrm{e}^{\mathrm{x}+\mathrm{i}}$ on complex numbers in polar coordinates, until we get the derivation of Euler's formula.
\end{abstract}

Keywords: Euler; formula; norm; argument.

\section{INTRODUCTION}

Euler's formula is widely used in solving mathematics or calculus, especially in solving complex numbers. Euler's formula is also used in The Exact Iterative Riemann Solver, The Approximate Riemann Solver of Roe, The HLLE Riemann Solver. To obtain this model, the authors use the derivation from $\lim _{x \rightarrow \infty}\left(1+\frac{1}{\mathrm{x}}\right)^{\mathrm{x}}=\mathrm{e}$.

In this derivation we substitute the norm and argument of $\mathrm{e}^{\mathrm{x}+\mathrm{iy}}$ on complex numbers in polar coordinates, until we get the derivation of Euler's formula..

\section{METHODS}

The inventor of the Euler formula is Leonhard Euler (1707 - 1783) $\mathrm{e}^{\mathrm{i} \theta}=\cos \theta+\mathrm{i} \sin \theta$, but the derivation of the formula is rarely stated clearly by mathematicians, therefore the author will convey in detail where the origin of the Euler formula is, namely by finding the length of the vector $\mathrm{e}^{\mathrm{x}+\mathrm{iy}}$ with the Limit method and the argument of $\mathrm{e}^{\mathrm{x}+\mathrm{iy}}$ using complex numbers in Polar coordinates.

\section{RESULTS AND DISCUSSION}

In this case, to get the derivation of Euler's formula, the writer describes the norm and argument of $\mathrm{e}^{\mathrm{x}+\mathrm{iy}}$.

First we find the norm of $\mathrm{e}^{\mathrm{x}+\mathrm{iy}}$.

We already know that $\lim _{x \rightarrow \infty}\left(1+\frac{1}{\mathrm{x}}\right)^{\mathrm{x}}=\mathrm{e}$

analog $\lim _{n \rightarrow \infty}\left(1+\frac{1}{\mathrm{n}}\right)^{\mathrm{n}}=\mathrm{e}$

so that $\lim _{n \rightarrow \infty}\left(1+\frac{\mathrm{x}}{\mathrm{n}}\right)^{\mathrm{n}}=\mathrm{e}^{\mathrm{x}}$,

or $\mathrm{e}^{\mathrm{x}}=\lim _{n \rightarrow \infty}\left(1+\frac{\mathrm{x}}{\mathrm{n}}\right)^{\mathrm{n}}$.

Given that the above formula applies to the complex number $\mathrm{z}$, then by substituting $\mathrm{x}=\mathrm{z}$,

$$
\text { we get } \mathrm{e}^{\mathrm{z}}=\lim _{n \rightarrow \infty}\left(1+\frac{\mathrm{z}}{\mathrm{n}}\right)^{\mathrm{n}},
$$

where $\mathrm{z}=\mathrm{x}+$ iy (complex numbers in Cartesian coordinates),

$$
\begin{aligned}
& \text { So that } \mathrm{e}^{\mathrm{x}+\mathrm{y}}=\lim _{n \rightarrow \infty}\left(1+\frac{\mathrm{x}+\mathrm{y}}{\mathrm{n}}\right)^{\mathrm{n}}, \\
& \mathrm{e}^{\mathrm{x}+\mathrm{y}}=\lim _{n \rightarrow \infty}\left[\left(1+\frac{\mathrm{x}}{\mathrm{n}}\right)+i \frac{y}{n}\right]^{\mathrm{n}} \ldots \ldots \ldots \ldots . . . . . . .
\end{aligned}
$$


Norm from $\mathrm{e}^{\mathrm{x}+\mathrm{iy}}$ is

$$
\begin{aligned}
& \left|\mathrm{e}^{\mathrm{x}+\mathrm{y}}\right|=\lim _{n \rightarrow \infty}\left[\sqrt{\left(1+\frac{\mathrm{x}}{\mathrm{n}}\right)^{2}+\left(\frac{y}{n}\right)^{2}}\right]^{\mathrm{n}}, \\
& \left|\mathrm{e}^{\mathrm{x}+\mathrm{i}}\right|=\lim _{n \rightarrow \infty}\left[\left(1+\frac{2 x}{n}+\frac{x^{2}}{n^{2}}+\frac{y^{2}}{n^{2}}\right)^{1 / 2}\right]^{\mathrm{n}}, \\
& \left|\mathrm{e}^{\mathrm{x}+\mathrm{y}}\right|=\lim _{n \rightarrow \infty}\left[1+\left(\frac{2 x}{n}+\frac{x^{2}+y^{2}}{n^{2}}\right)\right]^{1 / 2^{\mathrm{n}}}, \\
& \left|\mathrm{e}^{\mathrm{x}+\dot{\mathrm{y}}}\right|=e^{\lim _{n \rightarrow \infty}\left(\frac{2 x}{n}+\frac{x^{2}+y^{2}}{n^{2}}\right)(1 / 2 \mathrm{n})}, \\
& \left|\mathrm{e}^{\mathrm{x}+\mathrm{y}}\right|=e^{\lim _{n \rightarrow \infty}\left(x+\frac{x^{2}+y^{2}}{2 n}\right)}, \\
& \left|\mathrm{e}^{\mathrm{x}+\dot{\mathrm{y}}}\right|=e^{\lim _{n \rightarrow \infty}\left(x+\frac{x^{2}+y^{2}}{2 \infty}\right)}, \\
& \left|\mathrm{e}^{\mathrm{x}+\mathrm{y}}\right|=e^{\lim _{n \rightarrow \infty}(x+0)}, \\
& \text { So }\left|e^{x+y}\right|=e^{x}
\end{aligned}
$$

To find the argument of $\mathrm{e}^{\mathrm{x}+\mathrm{iy}}$, we first consider complex numbers in Polar coordinates $z=r(\cos \theta+\mathrm{i} \sin \theta)$, then $\operatorname{tg} \theta=\frac{\mathrm{y}}{\mathrm{x}}$, such that $\theta=\operatorname{arctg} \frac{\mathrm{y}}{\mathrm{x}}$

and $z^{n}=r^{n}(\cos \theta+\mathrm{i} \sin \theta)^{\mathrm{n}}$.

Where $(\cos \theta+\mathrm{i} \sin \theta)^{\mathrm{n}}=(\cos \mathrm{n} \theta+\mathrm{i} \sin \mathrm{n} \theta)$ according to De Moivre's Theorem,

then $z^{n}=r^{n}(\cos \mathrm{n} \theta+\mathrm{i} \sin \mathrm{n} \theta), \quad$ so that, or $\arg \left(z^{n}\right)=\mathrm{n} \operatorname{arctg} \frac{\mathrm{y}}{\mathrm{x}}$
Then we determine the argument of $\mathrm{e}^{\mathrm{x}+\mathrm{i} y}$, where from equation (1)

we have $\mathrm{e}^{\mathrm{x}+\mathrm{y}}=\lim _{n \rightarrow \infty}\left[\left(1+\frac{\mathrm{x}}{\mathrm{n}}\right)+i \frac{y}{n}\right]^{\mathrm{n}}$,

then

$$
\arg \left(\mathrm{e}^{\mathrm{x}+\mathrm{y}}\right)=\lim _{n \rightarrow \infty} \mathrm{n}\left(\operatorname{arctg} \frac{\frac{y}{n}}{\left(1+\frac{\mathrm{x}}{\mathrm{n}}\right)}\right),
$$

$\arg \left(\mathrm{e}^{\mathrm{x}+\mathrm{y}}\right)=\lim _{n \rightarrow \infty} \mathrm{n}\left(\operatorname{arctg} \frac{\frac{y}{n}}{\left(1+\frac{\mathrm{x}}{\mathrm{n}}\right)} \frac{n}{n}\right)$,

$\arg \left(\mathrm{e}^{\mathrm{x}+\mathrm{y}}\right)=\lim _{n \rightarrow \infty} \mathrm{n}\left(\operatorname{arctg} \frac{y}{n+x}\right)$

$\arg \left(\mathrm{e}^{\mathrm{x}+\dot{\mathrm{y}}}\right)=\lim _{n \rightarrow \infty} \mathrm{n}\left(\frac{\operatorname{arctg} \frac{y}{n+x}}{\frac{y}{n+x}}\right) \frac{y}{n+x}$.

Because

$\lim _{t \rightarrow \infty}\left(\frac{\operatorname{arctg} \frac{1}{t}}{\frac{1}{t}}\right)=\lim _{t \rightarrow \infty} \frac{\frac{1}{1+\left(\frac{1}{t}\right)^{2}}\left(\frac{1}{t}\right)^{\prime}}{\left(\frac{1}{t}\right)^{\prime}}=\lim _{t \rightarrow \infty} \frac{1}{1+\frac{1}{t^{2}}}=1$,

then $\arg \left(\mathrm{e}^{\mathrm{x}+\mathrm{i} y}\right)=\lim _{n \rightarrow \infty} \frac{y}{n+x}$, or $\arg \left(\mathrm{e}^{\mathrm{x}+\mathrm{y} y}\right)=\frac{y}{1}$.

So $\arg \left(\mathrm{e}^{\mathrm{x}+\mathrm{y}}\right)=y$

Complex numbers in Polar coordinates $z=r(\cos \theta+\mathrm{i} \sin \theta)$, where $r=|z|$ and $\theta=\arg (z)$, so that $z=|z|[\cos \{\arg (\mathrm{z})\}+\mathrm{i} \sin \{\arg (\mathrm{z})\}]$

Let $\mathrm{Z}=\mathrm{e}^{\mathrm{x}+\dot{\mathrm{y}}}$, then equation (4) will change to: $\mathrm{e}^{\mathrm{x}+\mathrm{y}}=\left|\mathrm{e}^{\mathrm{x}+\mathrm{y}}\right|\left[\cos \left\{\arg \left(\mathrm{e}^{\mathrm{x}+\mathrm{y}}\right)\right\}+\mathrm{i} \sin \left\{\arg \left(\mathrm{e}^{\mathrm{x}+\mathrm{y}}\right)\right\}\right]$ becomes:

Substitute (2) and (3) in (5), so that equation (5)

$$
\begin{aligned}
& \mathrm{e}^{\mathrm{x}+\mathrm{y}}=e^{x}(\cos y+\mathrm{i} \sin y), \\
& \mathrm{e}^{\mathrm{x}} \mathrm{e}^{\mathrm{i}}=e^{x}(\cos y+\mathrm{i} \sin y),
\end{aligned}
$$

So that $\mathrm{e}^{\mathrm{i}}=\cos y+\mathrm{i} \sin y$

Substitute $y=\theta$ in equation (6), then equation (6)

changes to : $\mathrm{e}^{\mathrm{i} \theta}=\cos \theta+\mathrm{i} \sin \theta$ 


\section{CONCLUSION}

In this decrease, a decrease has been carried out by searching first.

The Norm of $\mathrm{e}^{\mathrm{x}+\mathrm{iy}}$ is $\left|\mathrm{e}^{\mathrm{x}+\mathrm{y}}\right|=e^{x}$ and the argument of $\mathrm{e}^{\mathrm{x}+\mathrm{i}}$ is $\arg \left(\mathrm{e}^{\mathrm{x}+\mathrm{y}}\right)=y$, then by substituting for complex numbers in polar coordinates is $\mathrm{e}^{\mathrm{x}+\mathrm{y}}=\left|\mathrm{e}^{\mathrm{x}+\mathrm{y}}\right|\left[\cos \left\{\arg \left(\mathrm{e}^{\mathrm{x}+\mathrm{y}}\right)\right\}+\mathrm{i} \sin \left\{\arg \left(\mathrm{e}^{\mathrm{x}+\mathrm{y}}\right)\right\}\right]$

Derivation of the Euler's formula has been described above and obtained its derivation :

$$
\mathrm{e}^{\mathrm{i} \theta}=\cos \theta+\mathrm{i} \sin \theta
$$

where $\theta=\operatorname{arctg} \frac{\mathrm{y}}{\mathrm{x}}$.

\section{REFERENCES}

Anton, H., Rorres, C. (2014). Solutions for Elementary Linear Algebra: Applications Version 11th. Published simultaneously in Canada.

Bondan, A. (2001). Aljabar Linier. Jakarta: Penerbit Universitas Trisakti.

Briggs, W., Cochran, L., et al. (2018). Calculus: Early Transcendentals Third Edition. Pearson Published.

Cherney, D., Denton, T., Thomas, R., Waldron, A. (2013). Linear Algebra. Attribution-NonCommercial-ShareAlike 3.0 Unported.

Gazali, W. (2005). Matriks dan Transformasi Linear. Yogyakarta: Penerbit Graha Ilmu.

Gazali, W., Soedadyatmodjo (2005). Kalkulus. Yogyakarta: Penerbit Graha Ilmu.

Gazali, W., Soedadyatmodjo (2007). Kalkulus Edisi 2. Yogyakarta: Penerbit Graha Ilmu.

Hefferon, J. (2020). Linear Algebra with Applications, 4th edition. Vermont USA. : https://joshua.smevt. edu/linearalgebra/

L. Peterson, G., S. Sochacki, J. (2002). Linear Algebra and Differential Equationss. Addison Wesley Publishing Company.

Larson, R., H. Edwards, B. (2017). Calculus. CENGAGE Published.

Leon, S. (2020). Linear Algebra with Applications, 10th Edition. Published Pearson.

Madiana, A.M. (2000). Matriks dan Ruang Vektor Edisi Pertama. Yogyakarta: Penerbit Universitas Atma Jaya Yogyakarta.

Purcell, V., (2022). Calculus 9th Edition. Prentice Hall Published

STEWART, J. (2008). CALCULUS Early Transcendentals Sixth Edition. McMASTER UNIVERSITY: Thomson Brooks/ Cole.

Strang, G. (2011). Linear Algebra. Cambridge (USA) : MIT Press. 